\title{
Extraordinary Late\#Time Infrared Emission of Type IIn Supernovae
}

\section{Citation}

Gerardy, Christopher L., Robert A. Fesen, Ken'ichi Nomoto, Peter M. Garnavich, Saurabh Jha, Peter M. Challis, Robert P. Kirshner, Peter Hoflich, and J. Craig Wheeler. 2002. "Extraordinary Late\#Time Infrared Emission of Type IIn Supernovae." The Astrophysical Journal 575 (2): 100717. https://doi.org/10.1086/341430.

\section{Permanent link}

http://nrs.harvard.edu/urn-3:HUL.InstRepos:41399881

\section{Terms of Use}

This article was downloaded from Harvard University's DASH repository, and is made available under the terms and conditions applicable to Other Posted Material, as set forth at http:// nrs.harvard.edu/urn-3:HUL.InstRepos:dash.current.terms-of-use\#LAA

\section{Share Your Story}

The Harvard community has made this article openly available.

Please share how this access benefits you. Submit a story.

Accessibility 


\title{
EXTRAORDINARY LATE-TIME INFRARED EMISSION OF TYPE IIn SUPERNOVAE ${ }^{1}$
}

\author{
Christopher L. Gerardy, ${ }^{2}$ Robert A. Fesen, ${ }^{2}$ Ken' ichi Nomoto, ${ }^{3}$ Peter M. Garnavich, ${ }^{4}$ Saurabh Jha, ${ }^{5}$ \\ Peter M. Challis, ${ }^{5}$ Robert P. Kirshner, ${ }^{5}$ Peter Höflich, ${ }^{6}$ and J. Craig Wheeler 6 \\ Received 2001 December 20; accepted 2002 April 25
}

\begin{abstract}
Near-infrared observations are presented for five Type IIn supernovae (SN 1995N, SN 1997ab, SN 1998S, SN 1999Z, and SN 1999el) that exhibit strong infrared excesses at late times ( $t \gtrsim 100$ days). $H$ - and $K$-band emission from these objects is dominated by a continuum that rises toward longer wavelengths. The data are interpreted as thermal emission from dust, probably situated in a preexisting circumstellar nebula. The IR luminosities implied by single-temperature blackbody fits are quite large, $\geq 10^{41}-10^{42} \mathrm{ergs} \mathrm{s}^{-1}$, and the emission evolves slowly, lasting for years after maximum light. For SN 1995N, the integrated energy release via IR dust emission was $\approx 0.5-1 \times 10^{50}$ ergs. A number of dust heating scenarios are considered, the most likely being an infrared echo powered by X-ray and UV emissions from the shock interaction with a dense circumstellar medium.
\end{abstract}

Subject headings: circumstellar matter — dust, extinction — infrared: stars — supernovae: individual (SN 1995N, SN 1997ab, SN 1998S, SN 1999Z, SN 1999el)

\section{INTRODUCTION}

\subsection{Background}

On 1980 January 3, Merrill (1980) detected infrared (IR) emission from SN 1979C that was stronger toward longer wavelengths. Since then, similar "IR excesses" have been observed in a number of other supernovae, including $\mathrm{SN}$ 1980K (Telesco et al. 1981), SN 1982E (Graham et al. 1983; Graham \& Meikle 1986), SN 1982L, SN 1982R (Graham \& Meikle 1986), SN 1985L (Elias et al. 1986), SN 1993J (Lewis et al. 1994), SN 1994Y (Garnavich, Noriega-Crespo, \& Moro-Martin 1996), SN 1998S (Gerardy et al. 2000; Fassia et al. 2000), and SN 1997ab (Gerardy et al. 2000). These IR excesses have generally been interpreted as thermal emission from dust, either forming in the ejecta (Merrill 1980; Telesco et al. 1981; Dwek et al. 1983; Elias et al. 1986; Kozasa, Hasegawa, \& Nomoto 1989a, 1989b, 1991; Lucy et al. 1991; Colgan et al. 1994; Wooden et al. 1993) or lying in a preexisting circumstellar medium and heated by the supernova flash as an "IR echo" (Bode \& Evans 1980; Dwek 1983; Graham et al. 1983; Graham \& Meikle 1986; Lewis et al. 1994; Garnavich et al. 1996).

In the first scenario, dust forms in the cooling ejecta after it reaches a critical temperature. Late-time thermal emission from such dust has been invoked many times to explain near-infrared (NIR) excesses observed in nova outbursts (Gehrz 1988), where the critical formation temperature is typically $1000-1200 \mathrm{~K}$. While such a picture is perhaps the simplest explanation for the dust emission (e.g., SN 1987A: Kozasa et al. 1989a, 1989b, 1991; Danziger et al. 1991; Lucy

\footnotetext{
${ }^{1}$ Based in part on data collected at Subaru Telescope, which is operated by the National Astronomy Observatory of Japan.

2 Department of Physics and Astronomy, 6127 Wilder Laboratory, Dartmouth College, Hanover, NH 03755-3528.

${ }^{3}$ Department of Astronomy and Research Center for the Early Universe, University of Tokyo, Bunkyo-ku, Tokyo 113-0033, Japan.

${ }^{4}$ Department of Physics, University of Notre Dame, 225 Nieuwland Science Hall, Notre Dame, IN 46556.

${ }^{5}$ Harvard-Smithsonian Center for Astrophysics, 60 Garden Street, Cambridge, MA 02138.

${ }^{6}$ Department of Astronomy, University of Texas at Austin, Austin, TX 78712
}

et al. 1991), it can often be ruled out by energy considerations. Since the dust grain cooling time is short, and dust is localized in the ejecta where light-travel times are small, the IR luminosity must be limited by the energy available in the supernova at the time of observation. These sources include the thermal energy stored in the dusty gas, UV/X-ray radiation from the supernova, and gamma radiation from radioactive heavy elements in the ejecta. At late times $(t \geq 200$ days), the luminosity of observed IR excesses becomes increasingly difficult to explain within the dust formation scenario (Dwek 1983; Gerardy et al. 2000).

In light of this problem, a number of authors have suggested that the observed IR excesses are due to IR dust echoes. In this scenario, the emitting dust is not localized to the ejecta but is distributed in an extended circumstellar nebula. As the supernova flash propagates outward into the nebula, the dust is heated and then radiates thermally in the IR. Thus the late-time IR luminosity is powered by the maximum-light flash that, even after geometric dilution over a large sphere, is potentially a larger energy source than that available to dust in the $\mathrm{SN}$ ejecta.

Such energy considerations tended to make IR echoes a more attractive explanation for the observed IR excesses. For example, Dwek (1983) found that the IR emission from the Type IIL events SN 1979C and SN 1980K was too bright and that there was not enough energy in these objects at late times for a dust formation scenario. They were, however, able to fit the observed NIR excesses with an echo model. Similarly, Graham \& Meikle (1986) were able to use an IR echo model to reproduce the observed IR light curve of the untyped object SN 1982E.

On the other hand, Elias et al. (1986) observed IR emission in SN 1985L that became brighter before fading, in contradiction to IR echo models that predict a plateau followed by fading and only rise at very early times when the supernova is bright. Elias et al. (1986) argued that this was therefore a strong case for dust formation rather than an IR echo. However, Emmering \& Chevalier (1988) published models of echoes in aspherical dust clouds that showed that IR echoes from highly flattened dust distributions could exhibit more complicated rising and falling IR light curves. 
Much stronger evidence for dust formation was seen in SN 1987A, where increased IR emission was accompanied by a corresponding decrease in optical emission, while at the same time emission-line profiles were observed to shift toward the blue (McCray 1993 and references therein). Both effects argue in favor of dust formed with the supernova ejecta. Similarly, in SN 1998S, the evolution of the hydrogen and helium line profiles also suggested that dust formed in the ejecta as the redshifted side of the profile steadily faded, while the blueshifted side remained nearly constant (Gerardy et al. 2000). In this case, however, the thermal emission in the NIR was too bright to have come solely from the ejecta (Fassia et al. 2000), suggesting that there may be two different dust components in SN 1998S: newly formed dust in the ejecta and brightly emitting dust in the dense circumstellar medium (CSM).

\subsection{Recent Results on Type IIn Supernovae}

The NIR excesses observed in SN 1998S and SN 1997ab have added new dimensions to the problem (Gerardy et al. 2000; Fassia et al. 2000). Both objects are classified as Type IIn events, a subclass of Type II supernovae that exhibits multicomponent "Seyfert-like" hydrogen and helium emission lines (Schlegel 1990). This class is often associated with very slowly decaying light curves and strong X-ray emission (Filippenko 1997 and references therein). They are thought to be interacting with a dense and perhaps clumpy circumstellar wind resulting from significant mass loss prior to core collapse (Chugai \& Danziger 1994).

The IR emission in SN 1997ab and SN 1998S was quite luminous $\left(10^{40}-10^{41} \mathrm{ergs} \mathrm{s}^{-1}\right)$ at late epochs. For comparison, the excesses seen in SN 1979C and SN 1980K were first observed around day 200 and decayed exponentially with $e$ folding times of $\sim 100$ days. Yet the strong NIR excess in SN 1997ab was observed more than 3 yr after the explosion.

Large IR luminosities at late epochs strained both the dust formation and IR echo models, especially for SN 1997ab. Furthermore, the NIR spectra of SN 1997ab and SN 1998S were nearly identical, even though SN 1997ab was approximately 3 times older. This seemed to imply that either these events were coincidentally observed when they would show the same spectrum or the evolutionary timescale of these objects was quite long. These issues, plus the Type IIn classification of SN 1997ab and SN 1998S, led Gerardy et al. (2000) to propose that the IR emission was powered by the circumstellar interaction either directly through shock heating or via UV/X-ray emission from the shocked gas. They were unable to distinguish between circumstellar dust and newly formed ejecta dust as the emission source, but Fassia et al. (2000) used $K$ and $L^{\prime}$ photometry from an earlier epoch to rule out ejecta dust as the principal NIR emission source in SN 1998S.

In this paper, we present further NIR observations following the evolution of SN 1998 S and SN 1997ab. We also present NIR observations of three other Type IIn supernovae, SN 1995N, SN 1999Z, and SN 1999el, which also exhibit large late-time IR excesses. In $\S 2$, we discuss the observations and data reduction procedures. NIR spectral energy distributions (SEDs) are presented for all objects in $\S 3$, along with NIR spectra of SN $1995 \mathrm{~N}$ at four epochs covering nearly $5 \mathrm{yr}$ of evolution. We then consider several emission scenarios, including interaction shock precursor, shock-heated dust, simple IR echo, and interaction-powered echo models. We summarize our conclusions in $\S 4$.

\section{OBSERVATIONS}

\subsection{Supernova Dates and Distances}

SN 1995 N was discovered on 1995 May 5 by Pollas et al. (1995) in MCG -02-38-017. Based on optical spectra, Pollas et al. (1995) estimated that it was at least 10 months old, and following Fransson et al. (2002), we will adopt 1994 July 4 as the date of zero epoch.

SN 1998 S was discovered in NGC 3877 by Z. Wan (Li, Li, \& Wan 1998; Qiu 1998) on 1998 March 2. Following Gerardy et al. (2000), we chose the date of maximum light of 1998 March 18 (P. Garnavich 1998, private communication) as the date of zero epoch.

The other three supernovae were discovered either near or after maximum light, and we adopt the discovery date as zero epoch for these objects. SN 1997ab was discovered in an objective prism image taken on 1996 April 11 for the Hamburg Quasar Survey (Hagen, Engels, \& Reimers 1997). The host galaxy is the faint dwarf irregular galaxy HS 0948+2018.

SN $1999 Z$ was discovered on 1999 February 8 by Schwartz (1999) in UGC 5608, and SN 1999el was discovered in NGC 6951 by the Beijing Astronomical Observatory (BAO) Supernova Survey on 1999 October 20 (Cao et al. 1999).

Approximate distances to the supernovae were calculated from the recession velocities of the host galaxies, using $H_{0}=72 \mathrm{~km} \mathrm{~s}^{-1} \mathrm{Mpc}^{-1}$ (Freedman et al. 2001). For the three nearest objects, SN 1995N, SN1998S, and SN 1999el, recession velocities corrected for Virgocentric infall (taken from the Lyon-Meuden Extragalactic Database $)^{7}$ were used. The resulting distances are $26 \mathrm{Mpc}\left(1857 \mathrm{~km} \mathrm{~s}^{-1}\right), 15.5$ Mpc (1114 $\left.\mathrm{km} \mathrm{s}^{-1}\right)$, and $24 \mathrm{Mpc}\left(1705 \mathrm{~km} \mathrm{~s}^{-1}\right)$, respectively. For the more distant objects SN 1997ab and SN 1999Z, raw observed redshifts of $3600 \mathrm{~km} \mathrm{~s}^{-1}$ (Hagen \& Reimers 1997) and $15,100 \mathrm{~km} \mathrm{~s}^{-1}$ (Jha et al. 1999) were used, yielding distances of 50 and $210 \mathrm{Mpc}$, respectively.

\subsection{Near-Infrared Photometry}

NIR $J$-, $H$-, and $K$-band images of SN $1998 \mathrm{~S}, \mathrm{SN} 1997 \mathrm{ab}$, SN 1999Z, and SN 1999el were obtained from 1998 June to 2000 November using TIFKAM on the $2.4 \mathrm{~m}$ Hiltner telescope at MDM Observatory. TIFKAM is a high-throughput IR imager and spectrograph with a $512 \times 1024$ ALADDIN InSb detector. Dithered sets of on-target images were bracketed with images of nearby empty fields for sky subtraction. Most nights were photometric, and photometric solutions were achieved by observing Hubble Space Telescope (HST)/Two Micron All Sky Survey (2MASS) photometric standards (Persson et al. 1998) at a variety of air masses throughout the night. The data from nonphotometric nights were calibrated by matching the magnitudes of field stars to images from photometric nights.

$J$-, $H$-, and $K$-band images of SN $1995 \mathrm{~N}$ were taken during the period from 1996 July to 2001 May. The majority of observations were performed on the $1.2 \mathrm{~m}$ telescope at F. L.

\footnotetext{
${ }^{7}$ See http://leda.univ-lyon1.fr/.
} 
Whipple Observatory (FLWO) and with TIFKAM on the $2.4 \mathrm{~m}$ Hiltner telescope at MDM Observatory. Additional observations were obtained using the NASA Infrared Telescope Facility (IRTF), Apache Point Observatory (APO), and the Infrared Camera and Spectrograph (IRCS) on the Subaru Telescope. In order to avoid mixing data fluxed with different photometric systems, all SN 1995N observations were fluxed relative to several nearby field stars. A photometric solution for the field stars was obtained by observing Persson et al. (1998) standards from MDM. Thus, the SN $1995 \mathrm{~N}$ data are on the same photometric system as the data from the other four supernovae.

\subsection{Near-Infrared Spectroscopy}

NIR spectra of SN 1995N were observed from 1996 April to 2001 March. $H$ - and $K$-band spectra were obtained on 1996 April 4 and 5 using FSPEC on the Multiple-Mirror Telescope (MMT) and using the Infrared Cryogenic Spectrometer (CRSP) on the $4 \mathrm{~m}$ Mayall telescope at Kitt Peak National Observatory (KPNO) on 1997 June 16 and 1998 February 12. Each night, the MMT data were reduced separately and then combined into a single spectrum. An additional $K$-band spectrum of SN $1995 \mathrm{~N}$ was observed on 2001 March 5 using the IRCS (Kobayashi et al. 2000) on the Subaru Telescope. A log of all the NIR spectroscopy is presented in Table 1.

The spectroscopic observations were taken in many short exposures. Between exposures, the target was dithered across the slit to sample the chip at multiple locations and provide first-order background subtraction. Then onedimensional spectra were extracted using standard IRAF tasks. Wavelength calibration was achieved using $\mathrm{OH}$ lines in the night sky background for the MMT and KPNO spectra and using arc lamps for the Subaru data.

A nearby F5 V star from the Bright Star Catalog (Hoffleit \& Jaschek 1982) was used as a telluric standard for the MMT and KPNO data. Subaru data were corrected for telluric absorption using nearby A V and G V stars selected from the Gemini Spectroscopic Standard Star Catalogues. ${ }^{8}$ The $\mathrm{G}$ dwarf was divided by a solar spectrum (Livingston \& Wallace 1991; Wallace, Hinkle, \& Livingston 1993$)^{9}$ to remove stellar features. This was used to correct for telluric

\footnotetext{
${ }^{8}$ See http:/ /www.us-gemini.noao.edu/sciops/instruments/ niri/NIRIIndex.html.

${ }^{9}$ NSO Kitt Peak FTS data used here were produced by NSF/NOAO.
}

absorption in the A dwarf spectrum. The stellar features were fitted from the corrected A star spectrum and removed from the raw A star spectrum. The raw A star spectrum with the stellar features removed was then used to correct the target data for telluric absorption. (See Hanson, Conti, \& Rieke 1996 and Hanson, Rieke, \& Luhman 1998 for a more detailed discussion of this technique.) The instrumental response for all the runs was removed by matching the observed spectra of the telluric standards to model spectra from the stellar atmosphere calculations of Kurucz (1994).

The absolute flux levels of the spectra were set by matching the average flux in each passband to the NIR photometric data. For two of the epochs (days 1076 and 2435), photometric images were obtained within a few days and were used to set the flux. For the other two epochs, the NIR photometry was extrapolated using the two nearest photometric points for each band. In all cases, the passbands of Persson et al. (1998) were assumed. We estimate that the flux calibration is accurate to about $20 \%$.

There are a few issues that we have not accounted for thatcould slightly alter the results of our analysis. For example, the local reddening of the supernovae is not known for many of these objects, and thus no correction has been applied. We have also ignored the potential problems of exact photometric calibration of objects that have SEDs quite different from those of the photometric standards - in this case, very red continua in the $H$ and $K$ bands and strong line emission in the $J$ band. In light of these issues, we have emphasized the qualitative results from our analysis and treat the quantitative results as approximations rather than exact measurements.

\section{ANALYSIS}

Table 2 lists our IR photometric results. The observed magnitudes have been transformed into fluxes using the zero points of Beckwith et al. (1976) and Schultz et al. (2001). NIR SEDs are plotted for each of the supernovae in Figure 1. The SEDs have been corrected for galactic extinction using the measurements of Schlegel, Finkbeiner, \& Davis (1998) and assuming an $R_{V}=3.1$ extinction curve. Not all of the data for SN 1995N are plotted. Two epochs (days 1375 and 1785) had very poor $H$-band detections and are omitted in Figure 1, as are all epochs with only a $K$-band detection.

The overall trend is a slow evolution toward redder and fainter NIR continua. In three of the objects (SN 1998S,

TABLE 1

NEAR-INFRAREd SPECTROSCOPY OF SN 1995N

\begin{tabular}{|c|c|c|c|c|c|}
\hline Date & JD & $\begin{array}{l}\text { Epoch }^{\mathrm{a}} \\
\text { (days) }\end{array}$ & $\begin{array}{c}\text { Wavelength Coverage } \\
\qquad(\mu \mathrm{m})\end{array}$ & $\begin{array}{l}\text { Exposure } \\
\text { (s) }\end{array}$ & Observatory/Instrument \\
\hline \multirow[t]{2}{*}{1996 Apr 4 ......... } & \multirow[t]{2}{*}{$2,450,179$} & \multirow[t]{2}{*}{640} & $1.45-1.75$ & 1800 & \multirow[t]{2}{*}{ MMT + FSPEC } \\
\hline & & & $1.98-2.43$ & 3360 & \\
\hline \multirow[t]{2}{*}{1996 Apr 5 ......... } & \multirow[t]{2}{*}{$2,450,180$} & \multirow[t]{2}{*}{641} & $1.45-1.75$ & 3840 & \multirow[t]{2}{*}{ MMT + FSPEC } \\
\hline & & & $1.98-2.43$ & 1920 & \\
\hline \multirow[t]{2}{*}{1997 Jun 16 ........ } & \multirow[t]{2}{*}{$2,450,617$} & \multirow[t]{2}{*}{1078} & $1.45-1.73$ & 3600 & \multirow[t]{2}{*}{ KPNO 4 m + CRSP } \\
\hline & & & $1.96-2.49$ & 1200 & \\
\hline \multirow[t]{2}{*}{1998 Feb $12 \ldots \ldots}$. & \multirow[t]{2}{*}{$2,450,858$} & \multirow[t]{2}{*}{1319} & $1.44-1.72$ & 900 & \multirow{2}{*}{ KPNO 4 m + CRSP } \\
\hline & & & $1.92-2.50$ & 900 & \\
\hline 2001 Mar $5 . . . \ldots \ldots$. & $2,451,974$ & 2435 & $1.93-2.49$ & 3600 & Subaru + IRCS \\
\hline
\end{tabular}

a Adopted date for zero epoch is 1994 July 4. 
TABLE 2

NeAR-Infrared Photometry

\begin{tabular}{|c|c|c|c|c|c|c|}
\hline Date & $\mathrm{JD}$ & Epoch $^{\mathrm{a}}$ & $J$ & $H$ & $K$ & Observatory \\
\hline \multicolumn{7}{|c|}{ SN 1995N } \\
\hline 1996 Jul 4 ...... & $2,450,269$ & 730 & $16.90 \pm 0.03$ & $15.55 \pm 0.03$ & $13.41 \pm 0.04$ & IRTF \\
\hline 1997 Mar $25 . . . \ldots .$. & $2,450,533$ & 994 & $17.51 \pm 0.11$ & $15.90 \pm 0.05$ & $13.55 \pm 0.05$ & FLWO \\
\hline 1997 Apr 25......... & $2,450,564$ & 1025 & $17.35 \pm 0.13$ & $16.07 \pm 0.05$ & $13.61 \pm 0.07$ & FLWO \\
\hline 1997 Jun 12 ........ & $2,450,612$ & 1073 & $17.57 \pm 0.12$ & $15.99 \pm 0.07$ & $\ldots$ & APO \\
\hline 1997 Jun 15 ........ & $2,450,615$ & 1076 & $\ldots$ & $\ldots$ & $13.64 \pm 0.05$ & FLWO \\
\hline 1998 Mar $19 \ldots \ldots$. & $2,450,892$ & 1353 & $\ldots$ & $16.63 \pm 0.07$ & $14.21 \pm 0.04$ & FLWO \\
\hline 1998 Apr $10 \ldots \ldots \ldots$ & $2,450,914$ & 1375 & $>18.87$ & $17.29 \pm 0.16$ & $14.26 \pm 0.09$ & FLWO \\
\hline 1998 Jun 14 ........ & $2,450,979$ & 1440 & $\ldots$ & $16.70 \pm 0.10$ & $14.30 \pm 0.04$ & FLWO \\
\hline 1999 Feb $6 \ldots \ldots \ldots . .$. & $2,451,216$ & 1677 & $\ldots$ & $17.05 \pm 0.11$ & $14.82 \pm 0.07$ & FLWO \\
\hline 1999 May $29 . . . \ldots . .$. & $2,451,324$ & 1785 & $\ldots$ & $>18.14$ & $14.99 \pm 0.08$ & FLWO \\
\hline 2000 Apr $11 \ldots \ldots .$. & $2,451,646$ & 2107 & $19.57 \pm 0.23$ & $\ldots$ & $15.69 \pm 0.08$ & MDM \\
\hline 2000 Apr $12 \ldots \ldots \ldots$ & $2,451,647$ & 2108 & $\ldots$ & $18.15 \pm 0.12$ & 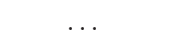 & MDM \\
\hline 2000 Apr $14 \ldots \ldots \ldots$ & $2,451,649$ & 2110 & $\ldots$ & & $15.85 \pm 0.10$ & FLWO \\
\hline $2001 \operatorname{Mar} 5 \ldots \ldots \ldots$ & $2,451,974$ & 2435 & $\ldots$ & $18.82 \pm 0.06$ & $16.31 \pm 0.06$ & Subaru \\
\hline 2001 Mar $30 \ldots \ldots . .$. & $2,452,000$ & 2461 & $\ldots$ & $\ldots$ & $16.41 \pm 0.05$ & MDM \\
\hline 2001 May 1 ......... & $2,452,032$ & 2493 & $\ldots$ & $\ldots$ & $16.47 \pm 0.14$ & MDM \\
\hline \multicolumn{7}{|c|}{ SN 1997ab } \\
\hline 1999 Mar $9 . \ldots \ldots \ldots$ & $2,451,248$ & 1062 & $17.58 \pm 0.05$ & $15.67 \pm 0.03$ & $13.86 \pm 0.04$ & MDM \\
\hline 2000 May $18 . . . . . . . .$. & $2,451,684$ & 1498 & $\ldots$ & $17.96 \pm 0.13$ & $15.54 \pm 0.04$ & MDM \\
\hline 2000 Nov $26 \ldots \ldots .$. & $2,451,876$ & 1690 & $\ldots$ & $19.30 \pm 0.18$ & $16.48 \pm 0.06$ & MDM \\
\hline \multicolumn{7}{|c|}{ SN 1998S } \\
\hline 1998 Jun $20 \ldots \ldots \ldots$ & $2,450,986$ & 94 & $14.37 \pm 0.03$ & $14.07 \pm 0.05$ & $13.46 \pm 0.07$ & MDM \\
\hline 1998 Oct $31 \ldots \ldots .$. & $2,451,119$ & 227 & $16.29 \pm 0.05$ & $14.83 \pm 0.04$ & $13.50 \pm 0.04$ & MDM \\
\hline 1998 Dec $3 \ldots \ldots \ldots . .$. & $2,451,152$ & 260 & $16.74 \pm 0.08$ & $15.05 \pm 0.06$ & $13.62 \pm 0.07$ & MDM \\
\hline 1999 Mar $8 \ldots \ldots \ldots . .$. & $2,451,247$ & 355 & $17.26 \pm 0.07$ & $15.46 \pm 0.04$ & $13.57 \pm 0.02$ & MDM \\
\hline 1999 Mar $9 . . . \ldots \ldots . .$. & $2,451,248$ & 356 & $17.28 \pm 0.08$ & $15.46 \pm 0.03$ & $13.59 \pm 0.03$ & MDM \\
\hline 2000 May $22 \ldots \ldots . .$. & $2,451,688$ & 796 & $\ldots$ & $17.57 \pm 0.19$ & $15.38 \pm 0.09$ & MDM \\
\hline \multicolumn{7}{|c|}{ SN 1999Z } \\
\hline 1999 Dec $1 \ldots \ldots \ldots . .$. & $2,451,515$ & 296 & $17.71 \pm 0.05$ & $\ldots$ & $16.49 \pm 0.05$ & MDM \\
\hline 2000 May $18 \ldots \ldots .$. & $2,451,684$ & 465 & $18.25 \pm 0.08$ & $\ldots$ & $16.07 \pm 0.07$ & MDM \\
\hline 2000 Nov $23 \ldots \ldots .$. & $2,451,873$ & 654 & $>20.11$ & $19.55 \pm 0.23$ & $16.84 \pm 0.06$ & MDM \\
\hline \multicolumn{7}{|c|}{ SN 1999el } \\
\hline 2000 May $18 \ldots \ldots .$. & $2,451,684$ & 198 & $18.33 \pm 0.15$ & & $16.29 \pm 0.08$ & MDM \\
\hline 2000 Nov $23 \ldots \ldots .$. & $2,451,873$ & 387 & $>18.99$ & $>18.53$ & $16.84 \pm 0.12$ & MDM \\
\hline
\end{tabular}

SN 1999Z, and SN 1999el), we observe the onset of the NIR excess. For example, in SN 1998S, the $K$-band flux remains essentially constant from days 94 to 355 , while the $J$ and $H$ bands fade as if the blue photospere fades to reveal a much more slowly evolving red continuum underneath. In contrast, the $K$-band flux increases during the blue-to-red transition in SN 1999Z, suggesting that the IR continuum might still have been rising during this period. SN 1995N and SN $1997 \mathrm{ab}$ already exhibited strong NIR excesses when they were first observed. During the observed period, SN 1997ab slowly evolved to redder colors, while SN 1995N exhibited virtually no color evolution over nearly 1800 days.

\subsection{Single-Temperature Fits}

For data showing clear IR excesses, we fit $H-K$ color temperatures with dust emission/absorption efficiencies $Q_{\lambda}=$ constant, $Q_{\lambda} \propto \lambda^{-1}$, and $Q_{\lambda} \propto \lambda^{-2}$. The results are listed in Table 3. We note that single-temperature dust emission is probably not a good description for the IR excesses, which would generally be a superposition of many dust temperatures. (It would be a valid description if the emitting dust were confined to a thin shell or very far away from the SN light source, so that $r^{-2}$ variations would be small.) However, since we have only NIR data, it is not possible to constrain the long-wavelength dust emission spectrum. Thus, our data are really sensitive to only the hottest components of the dust emission.

The best-fit curves for $Q_{\lambda} \propto \lambda^{-1}$ are shown in Figure 1 . We note that the $J$-band flux always lies significantly above the continuum fitted by the $H-K$ color. IR dust emission is only a very small component of the $J$-band flux, which is likely dominated by the $\mathrm{Pa} \beta$ emission line. As a result, we ignored the $J$-band flux in the analysis of the dust emission. Also, while we have plotted the $Q_{\lambda} \propto \lambda^{-1}$ curves, the $Q_{\lambda}=$ constant and $Q_{\lambda} \propto \lambda^{-2}$ curves also fit the $H$ - and $K$ - 


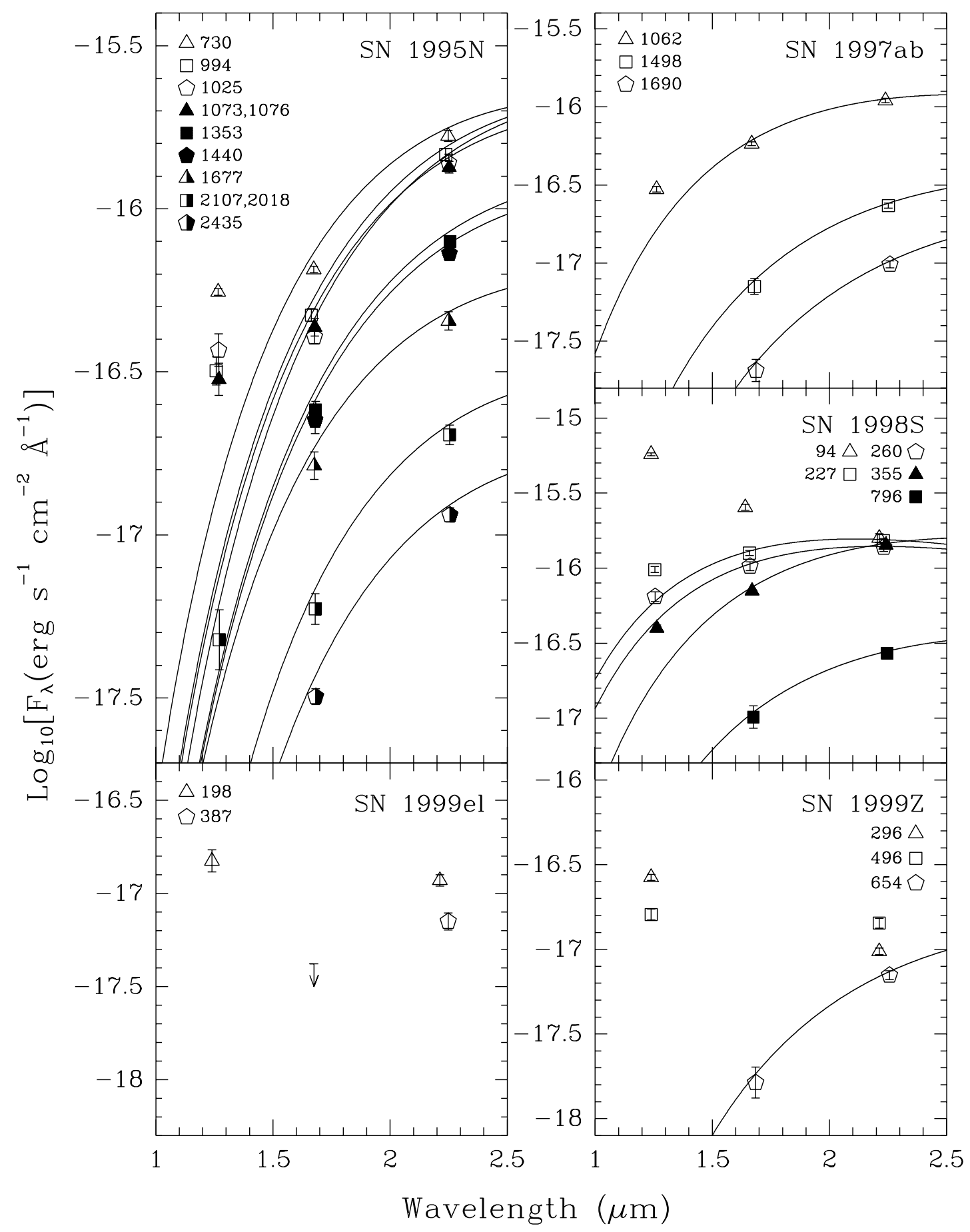

FIG. 1.-SEDs for SN 1995N, SN 1997ab, SN 1998S, SN 1999Z, and SN 1999el. The curves are the best-fit single-temperature blackbody curves for the observed $H-K$ colors, assuming a $\lambda^{-1}$ absorption/emission efficiency.

band spectra of SN 1995N. Without longer wavelength observations, we cannot constrain the dust absorption/ emission efficiency. For the rest of the analysis, we assume $Q_{\lambda} \propto \lambda^{-1}$ unless otherwise noted.

The observed $H-K$ temperature evolution of SN 1995N, SN 1997ab, and SN 1998S is plotted in the top panels of Figure 2. SN 1995N exhibits almost no temperature evolution, cooling only from about 900 to about $800 \mathrm{~K}$ between the first and last epochs. In contrast, the other two supernovae show significant color evolution. SN 1997ab appears to cool relatively linearly from 930 to $665 \mathrm{~K}$ over 600 days, while SN 1998S cooled quickly from 1140 to 900 K over 128 days (between days 227 and 355) and then more slowly down to $810 \mathrm{~K}$ over the next year. Apparently, the observation of nearly identical spectra/temperatures in SN 1998S and SN 1997ab noted by Gerardy et al. (2000) was merely a coincidence, as these objects are clearly evolving, albeit over a longer time period than the previously observed NIR excesses in supernovae.

The total IR fluxes, calculated by integrating the $H-K$ temperature fits over all wavelengths, are also listed in Table 3 and plotted in the bottom panels of Figure 2. These lumi- 
TABLE 3

Single-Temperature Fits

\begin{tabular}{|c|c|c|c|c|c|c|}
\hline \multirow[b]{2}{*}{$\begin{array}{l}\text { EPOCH } \\
\text { (days) }\end{array}$} & \multicolumn{2}{|c|}{$Q_{\lambda} \propto 1$} & \multicolumn{2}{|c|}{$Q_{\lambda} \propto \lambda^{-1}$} & \multicolumn{2}{|c|}{$Q_{\lambda} \propto \lambda^{-2}$} \\
\hline & $\begin{array}{c}T_{d} \\
(\mathrm{~K})\end{array}$ & $\begin{array}{c}L_{d} \\
\left(10^{40} \mathrm{ergs} \mathrm{s}^{-1}\right)^{\mathrm{a}}\end{array}$ & $\begin{array}{c}T_{d} \\
(\mathrm{~K})\end{array}$ & $\begin{array}{c}L_{d} \\
\left(10^{40} \mathrm{ergs} \mathrm{s}^{-1}\right)^{\mathrm{a}}\end{array}$ & $\begin{array}{c}T_{d} \\
(\mathrm{~K})\end{array}$ & $\begin{array}{c}L_{d} \\
\left(10^{40} \mathrm{ergs} \mathrm{s}^{-1}\right)^{\mathrm{a}}\end{array}$ \\
\hline \multicolumn{7}{|c|}{ SN 1995N } \\
\hline $730 \ldots \ldots \ldots \ldots \ldots$ & $930 \pm 20$ & $89 \pm 8$ & $830 \pm 15$ & $65 \pm 5$ & $745 \pm 15$ & $52 \pm 4$ \\
\hline 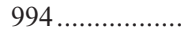 & $860 \pm 25$ & $101 \pm 12$ & $770 \pm 20$ & $71 \pm 8$ & $700 \pm 15$ & $55 \pm 5$ \\
\hline $1025 \ldots \ldots \ldots \ldots \ldots$ & $830 \pm 30$ & $108 \pm 19$ & $745 \pm 25$ & $75 \pm 11$ & $680 \pm 20$ & $57 \pm 8$ \\
\hline $1073,1076 \ldots \ldots$ & $860 \pm 30$ & $92 \pm 13$ & $775 \pm 20$ & $65 \pm 8$ & $700 \pm 20$ & $50 \pm 6$ \\
\hline $1353 \ldots \ldots \ldots \ldots . . .$. & $840 \pm 25$ & $59 \pm 8$ & $755 \pm 20$ & $41 \pm 5$ & $685 \pm 20$ & $32 \pm 4$ \\
\hline $1440 \ldots \ldots \ldots \ldots \ldots$ & $845 \pm 40$ & $53 \pm 10$ & $760 \pm 30$ & $37 \pm 6$ & $690 \pm 25$ & $29 \pm 4$ \\
\hline $1677 \ldots \ldots \ldots \ldots . . . . . . .$. & $900 \pm 50$ & $27 \pm 6$ & $805 \pm 40$ & $19 \pm 4$ & $725 \pm 35$ & $15 \pm 3$ \\
\hline $2107,2108 \ldots \ldots$ & $830 \pm 45$ & $16 \pm 4$ & $745 \pm 40$ & $11 \pm 3$ & $680 \pm 30$ & $8 \pm 2$ \\
\hline $2435 \ldots \ldots \ldots \ldots \ldots$ & $815 \pm 25$ & $10 \pm 2$ & $735 \pm 20$ & $7 \pm 1$ & $670 \pm 15$ & $5 \pm 0.6$ \\
\hline \multicolumn{7}{|c|}{ SN 1997ab } \\
\hline $1062 \ldots \ldots \ldots \ldots . . . .$. & $1060 \pm 20$ & $156 \pm 11$ & $930 \pm 15$ & $120 \pm 7$ & $825 \pm 15$ & $100 \pm 5$ \\
\hline 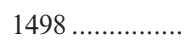 & $835 \pm 50$ & $65 \pm 14$ & $750 \pm 40$ & $45 \pm 9$ & $685 \pm 30$ & $35 \pm 6$ \\
\hline $1690 \ldots \ldots \ldots \ldots \ldots$ & $730 \pm 55$ & $48 \pm 17$ & $665 \pm 45$ & $31 \pm 10$ & $610 \pm 40$ & $22 \pm 6$ \\
\hline \multicolumn{7}{|c|}{ SN 1998S } \\
\hline $227 \ldots \ldots \ldots \ldots \ldots$ & $1350 \pm 45$ & $15 \pm 0.8$ & $1140 \pm 35$ & $12 \pm 0.5$ & $990 \pm 25$ & $11 \pm 0.4$ \\
\hline $260 \ldots \ldots \ldots \ldots \ldots$ & $1275 \pm 70$ & $14 \pm 1.5$ & $1085 \pm 52$ & $11 \pm 1$ & $950 \pm 40$ & $10 \pm 0.8$ \\
\hline $355 \ldots \ldots \ldots \ldots \ldots \ldots$ & $1025 \pm 15$ & $21 \pm 0.9$ & $905 \pm 10$ & $16 \pm 0.6$ & $805 \pm 10$ & $13 \pm 0.4$ \\
\hline $796 \ldots \ldots \ldots \ldots \ldots$ & $910 \pm 100$ & $5.5 \pm 1.9$ & $810 \pm 75$ & $4.0 \pm 1$ & $730 \pm 60$ & $3.1 \pm 0.8$ \\
\hline \multicolumn{7}{|c|}{ SN 1999Z } \\
\hline $654 \ldots \ldots \ldots \ldots \ldots \ldots$ & $760_{-55}^{+80}$ & $515_{-195}^{+235}$ & $690_{-45}^{+65}$ & $340_{-115}^{+135}$ & $630_{-35}^{+55}$ & $250_{-80}^{+90}$ \\
\hline \multicolumn{7}{|c|}{ SN 1999el } \\
\hline $387 \ldots \ldots \ldots \ldots \ldots . . .$. & $\lesssim 1245$ & $\gtrsim 2$ & $\lesssim 1060$ & $\gtrsim 1$ & $\lesssim 890$ & $\gtrsim 1$ \\
\hline
\end{tabular}

a Quoted sigmas do not include distance uncertainty.

nosities should be regarded as lower limits on the total IR luminosities, as the NIR is only sensitive to the hottest dust, and consequently, a significant flux from much colder dust could be missed in our data.

Although SN 1995N showed little temperature evolution, there is significant evolution of its IR luminosity. For the first four epochs, the luminosity seems relatively constant at about $7 \times 10^{41} \mathrm{ergs} \mathrm{s}^{-1}$. Then, after day 1000 , the luminosity appears to decline exponentially with an $e$-folding time of $\approx 600$ days. While no plateau phase was observed in SN $1997 \mathrm{ab}$, the IR luminosity also appears to decay exponentially, in this case with an $e$-folding time of $\approx 450$ days. However, the NIR behavior of SN 1998S was somewhat different. The inferred IR luminosity of SN 1998S increased $\sim 50 \%$ between days 260 and 355 and then faded between days 355 and 796 at a rate similar to that of SN 1997ab.

While we interpret the IR continuum as thermal emission from dust, it is important to estimate the extent that freefree emission might contribute to the NIR continuum, as there are strong recombination lines observed in these supernovae. Fransson et al. (2002) found that in SN 1995N, $\mathrm{H} \alpha$ was $\approx 8$ times stronger than expected for standard $\mathrm{H}_{\mathrm{I}}$ recombination because of collisional excitation and a high optical depth for $\mathrm{H} \alpha$. However, the higher order Balmer lines were consistent with case B recombination. Assuming standard nebular conditions and case $\mathrm{B}$ recombination, the
$\mathrm{H} \beta$ emission per unit volume is $4 \pi j_{\mathrm{H} \beta} \approx 1.2 \times 10^{-25} n^{2}$ ergs $\mathrm{cm}^{-3} \mathrm{~s}^{-1}$ (Osterbrock 1989). Using an abundance ratio of $\mathrm{He}$ to $\mathrm{H}$ of 0.35 by number (Fransson et al. 2002), the freefree emission per unit volume $\epsilon_{\mathrm{ff}} \approx 3.2 \times 10^{-27} T^{1 / 2} n^{2} \mathrm{ergs}$ $\mathrm{cm}^{-3} \mathrm{~s}^{-1}$ (Rybicki \& Lightman 1979), where we have assumed a velocity-averaged Gaunt factor $\bar{g}_{B} \approx 1.2$. For $T=10^{4} \mathrm{~K}$, this implies that $\epsilon_{\mathrm{ff}} \approx 3 \epsilon_{\mathrm{H} \beta}$. Fransson et al. (2002) report an $\mathrm{H} \beta$ flux of $8.8 \times 10^{-15} \mathrm{ergs} \mathrm{s}^{-1} \mathrm{~cm}^{-2}$ from SN $1995 \mathrm{~N}$ on day 718 , which translates to a free-free luminosity of $L_{\mathrm{ff}} \approx 2 \times 10^{39} \mathrm{ergs} \mathrm{s}^{-1}$. Compared to the IR luminosity on day 730 of $8 \times 10^{41} \mathrm{ergs} \mathrm{s}^{-1}$, the free-free continuum is down by 2 orders of magnitude and thus does not contribute significantly to the observed NIR continuum.

\subsection{Comparison to $X$-Ray Emission}

Comparison of the observed X-ray and IR light curves can be used to provide clues to the origin of the IR emission. Both SN 1995N (Fox et al. 2000) and SN 1998S (Pooley et al. 2002) have been detected in X-rays, and the observed $\mathrm{X}$-ray luminosities are plotted as stars in Figure 2.

One possible dust heating scenario is that the IR emission is from a precursor region in the CSM just ahead of the shock front that is being heated by X-rays from the postshock gas. In such a situation in which the light-travel time 


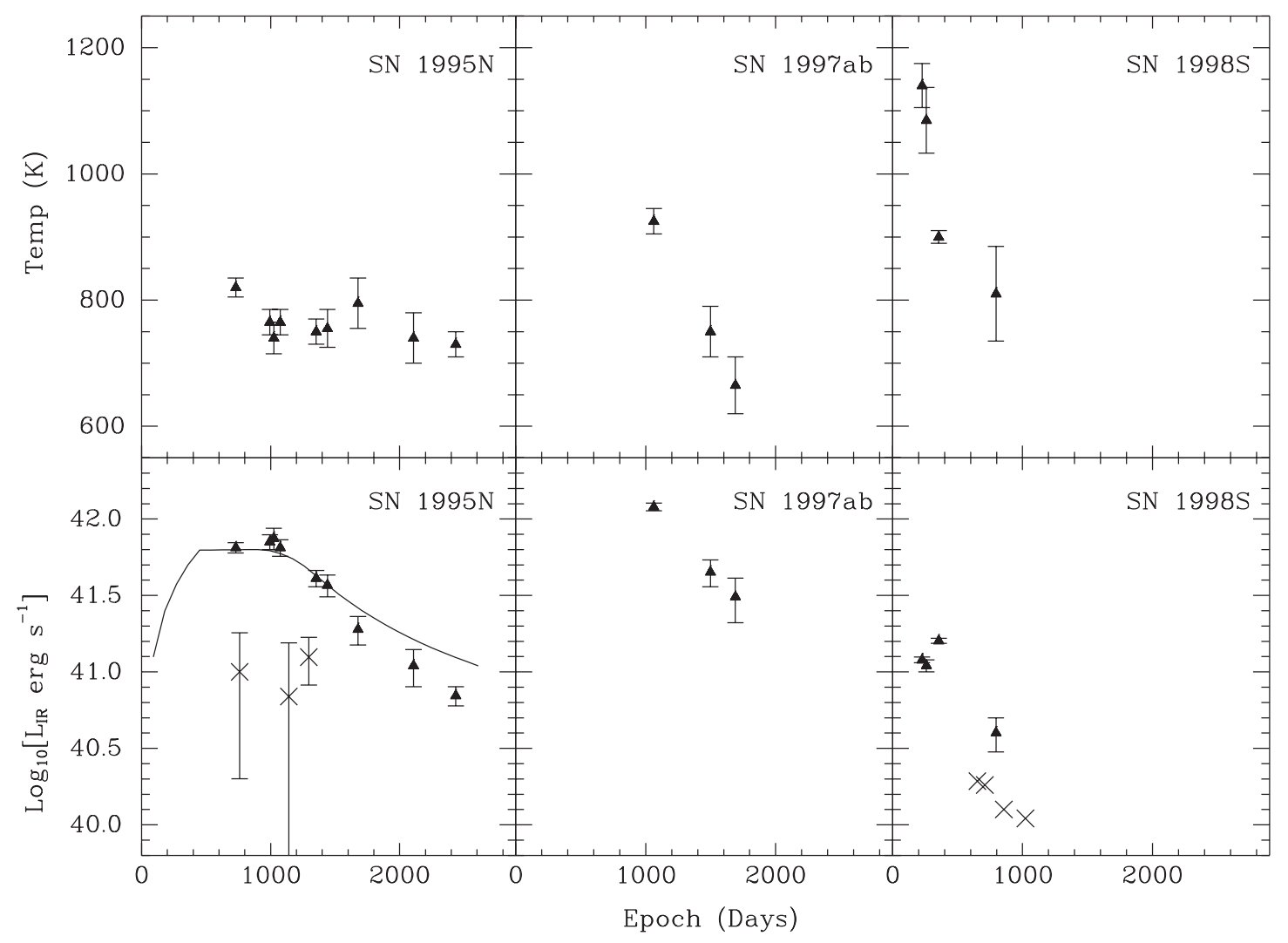

FIG. 2.-Top panels show the observed $H-K$ temperature evolution for SN 1995N, SN 1997ab, and SN 1998S, assuming a $\lambda^{-1}$ absorption/emission efficiency for the dust. The bottom panels plot the inferred IR luminosities from the $H-K$ temperature fits (triangles) and observed X-ray luminosities (crosses). The solid line in the bottom SN $1995 \mathrm{~N}$ plot is the IR luminosity from an IR echo model with a short exponential flash as the input supernova light curve.

is small, we might expect the X-ray luminosity and the IR luminosity to be well correlated. What we find, however, is that the IR evolution does not follow the X-ray light curve in SN 1995N, as the X-ray flux shows no signs of fading nearly 200 days after the IR begins to fall. Furthermore, the IR flux is significantly greater (as much as a factor of 5) than the X-rays. This would require that the actual emitted X-ray flux is much larger and that nearly all of the $\mathrm{X}$-rays are being absorbed by the circumstellar dust and reemitted in the IR. However, Fransson et al. (2002) found no indication of strong reddening in the optical spectra of SN 1995N, suggesting that the dust extinction in the CSM is low. These problems argue against an IR precursor interpretation.

We note that large IR/X-ray flux ratios are seen in supernova remnants with observed ratios varying from $\sim 2$ to $\sim 100$ (Dwek et al. 1987). In remnants, the IR emission is from hot, dusty, shocked gas, where thermal emission from collisionally heated dust can be the dominant cooling mechanism. However, the IR emission from supernova remnants is typically much cooler, and it seems unlikely that a significant amount of dust could be collisionally heated up to 800 $1000 \mathrm{~K}$ without destroying the dust grains. Collisional heating of dust to $\sim 1000 \mathrm{~K}$ would also require prohibitively large ion densities (greater than $10^{21} \mathrm{~cm}^{-3}$ for $T_{e}=10^{7} \mathrm{~K}$; N. Evans 2002, private communication). Furthermore, while there could be some time lag between changes in the $\mathrm{X}$-ray flux and changes in the IR flux, we would not expect to see the luminosity drop in the IR before it does in the X-rays, as seen in SN 1995N.

\subsection{Infrared Echo Models}

On the other hand, the shape of the SN 1995N IR light curve is similar to the luminosity evolution of simple IR echo models. A number of authors have presented models describing the IR echoes of supernovae in spherically symmetric dust distributions (Bode \& Evans 1980; Dwek 1983; Graham et al. 1983; Graham \& Meikle 1986). In this paper, we adopt the formalism of Emmering \& Chevalier (1988, hereafter EC88), which adds the generality of nonspherical flattened dust distributions. A common feature of spherical models is that they predict an IR light curve that rises quickly (only while the supernova is at its brightest) and then levels off in a plateau before finally decaying.

The light-curve plateau results because the supernova flash clears out a large dust-free cavity in the center of the circumstellar nebula by vaporizing all the dust. At the edge of this cavity is the hottest and brightest dust emission. Outside the cavity, the dust is cooler and fainter as the supernova flash is spread out over a larger surface. For a pulse (delta function) supernova light curve that explodes at time $t^{\prime}$, the observed flux at any time $t$ will come from dust lying on a paraboloid described by $t=t^{\prime}+r / c(1-\cos \theta)$. As time proceeds, the paraboloids become wider, and the vertex recedes from the supernova. At time $t_{v}=2 r_{v} / c$, the vertex of the paraboloid will reach the edge of the edge of the cavity. Before this time, the IR luminosity, dominated by emission from the cavity's edge, will be nearly constant, forming the plateau in the IR light curve. After the para- 
boloid leaves the cavity, the emission will come from the fainter and cooler dust, and thus the IR luminosity will fade. A plateau is also expected in models with more realistic $\mathrm{SN}$ light curves as long as the $\mathrm{SN}$ fades in significantly less time than it takes for the echo paraboloid to reach the cavity wall.

The observed IR light curve for SN $1995 \mathrm{~N}$ is at least qualitatively similar to the light curves of spherically symmetric IR echoes. However, the evolution timescale for SN $1995 \mathrm{~N}$ is much longer than for the models that were used to fit IR echoes to previous IR excesses in supernovae. In SN 1979C, SN 1980K, and SN 1982E, the IR excesses were detected 100-200 days after maximum light, and the IR light curve was already declining. The time $t_{v}$ at which the reemission paraboloids reached the cavity wall in the model fits was typically $\sim 50-100$ days. For SN $1995 \mathrm{~N}$, this would have to be more like 1000 days, where the observed IR light curve begins to decay rapidly. This timescale is set by the radius of the dust-free cavity, which scales as the square root of the supernova luminosity. Thus, the much longer timescale would imply that either the dust around SN $1995 \mathrm{~N}$ is much easier to vaporize or SN $1995 \mathrm{~N}$ is much brighter $(\sim 100 \times)$ than those other objects. A more likely solution might be that some event prior to core collapse cleared out the cavity.

\subsubsection{Numerical Calculations}

To test the IR echo scenario, we have calculated the total IR luminosity evolution and NIR spectra for echo models based on EC88. These models assume an axisymmetric dust distribution in which the isodensity curves are ellipsoids of revolution in which the dust distribution has a $b^{-2}$ radial dependence. The circumstellar dust is assumed to have an emission/absorption efficiency with a power-law wavelength dependence $Q_{\lambda} \propto \lambda^{-n}$. The model dust cloud has an inner dust-free zone, as discussed above, and has no sharp outer edge. The models implicitly assume that the optical depth of the dust cloud is low and do not account for absorption inside the circumstellar dust nebula, either between the SN and the heated dust or after reemission. Our calculations for the total IR luminosity $F_{\mathrm{IR}}(t)$ and the IR spectrum $F_{\lambda}(t)$ were performed by numerically integrating equations (8) and (15) of EC88.

The IR dust echo models have a number of parameters that can be varied. These include the dust emission/ absorption efficiency exponent $n$, the size of the inner cavity $r_{v}$, the dust temperature at the inner surface of the cavity $T_{v}$, the SN light curve, the degree to which the dust distribution is flattened, and for nonspherical dust distributions, the angle between the axis of symmetry and the line of sight. Since the observed plateau in the IR light curve was suggestive of a spherically symmetric dust distribution, we used spherically symmetric echo models for SN 1995N. This eliminates two of the model parameters and also makes the numerical calculations easier.

In principle, if the dust-free cavity were created by the supernova flash vaporizing the innermost dust, the cavity radius $r_{v}$, the peak luminosity of the supernova, and the dust temperature at the inner edge of the cavity $T_{v}$ would all be related. If the dust has a vaporization temperature $T_{v}$, then $r_{v}$ would be the radius at which the maximum luminosity of the supernova would heat the dust to $T_{v}$. In practice, however, a number of other details about the dust distribution must be known to relate these parameters (e.g., grain size, composition, and absolute values of the absorption/ emission efficiency and the dust density). Furthermore, if the cavity is the result of preexplosion evolution and not vaporization due to the $\mathrm{SN}$ flash, then no relationship would exist. Therefore, we treat $T_{v}$ and $r_{v}$ as independent parameters.

It is convenient to parameterize the cavity size by $t_{v}=2 r_{v} / c$, the epoch at which we first observe light reemitted by dust on the cavity surface directly behind the supernova along the line of sight. This parameter sets the timescale for the IR evolution. For models in which the supernova light curve decays quickly compared to this timescale, $t_{v}$ is near the "kink" in the IR light curve, where the plateau ends and the dust emission begins to decay. For SN $1995 \mathrm{~N}$, the kink in the IR light curve occurs near $t=1000$ days (see Fig. 2), so we consider models with $t_{v}$ near this epoch.

\subsubsection{Short-Flash Echo Model}

Since we have more data on SN $1995 \mathrm{~N}$ than on the other four Type IIn SNe, we use this event to test the IR echo scenario. The solid line in the bottom left panel of Figure 2 shows the IR light curve from a model in which the input $\mathrm{SN}$ light curve is a short flash compared to the echo timescale $t_{v}$. For the model shown, the SN light curve was exponentially decaying with an $e$-folding time $\Gamma=t_{v} / 10$ and dust emission/absorption exponent $n=1$. For short-flash models in which the supernova light curve fades much faster than $t_{v}$, the results are quite insensitive to the exact details of the input light curve, and a short "top-hat" light curve would have produced essentially the same results.

The model IR luminosity was matched to the observed plateau luminosity with $t_{v}$ scaled to 950 days to match the observed kink in the IR light curve. As can be seen in Figure 3, the resulting light curve is qualitatively similar to the observed IR luminosity evolution of SN 1995N, but it fades more slowly after the kink. However, this is not a strong test of the dust echo model. The "observed" IR luminosity is an extrapolation of the single-temperature continuum fit to the $H-K$ color and thus is only sensitive to the hottest dust. In contrast, the model light curve shows emission from a superposition of dust over a wide range of temperatures. As the echo ages and the emission comes from farther out in the nebula, the IR emission becomes more and more dominated by cooler dust that is missed by our NIR photometry.

A better test is to directly compare the observed fluxcalibrated spectra to the evolution of the model spectrum. The echo model calculates spectra for each epoch in dimensionless wavelength units $\lambda / \lambda_{v}$, where $\lambda_{v} \equiv k T_{v} / h$. To make a fit for a given set of parameters, the model spectrum is matched to a single epoch of the data. The model is shifted vertically to match the flux levels and horizontally to match the shape of the observed spectrum, essentially fitting $T_{v}$. The same shifts are then applied to the other epochs and the resulting spectral evolution can be compared to the data.

The results of such a procedure are plotted in Figure 3. In the top panel, we plot the observed spectra of SN $1995 \mathrm{~N}$ with spectra from the short-flash echo model for the corresponding epochs. The model luminosity and $T_{v}$ have been fitted to the second spectrum on day 1078 . The cavity time $t_{v}$ for this model is 950 days, and $T_{v} \approx 1100 \mathrm{~K}$. The resulting 


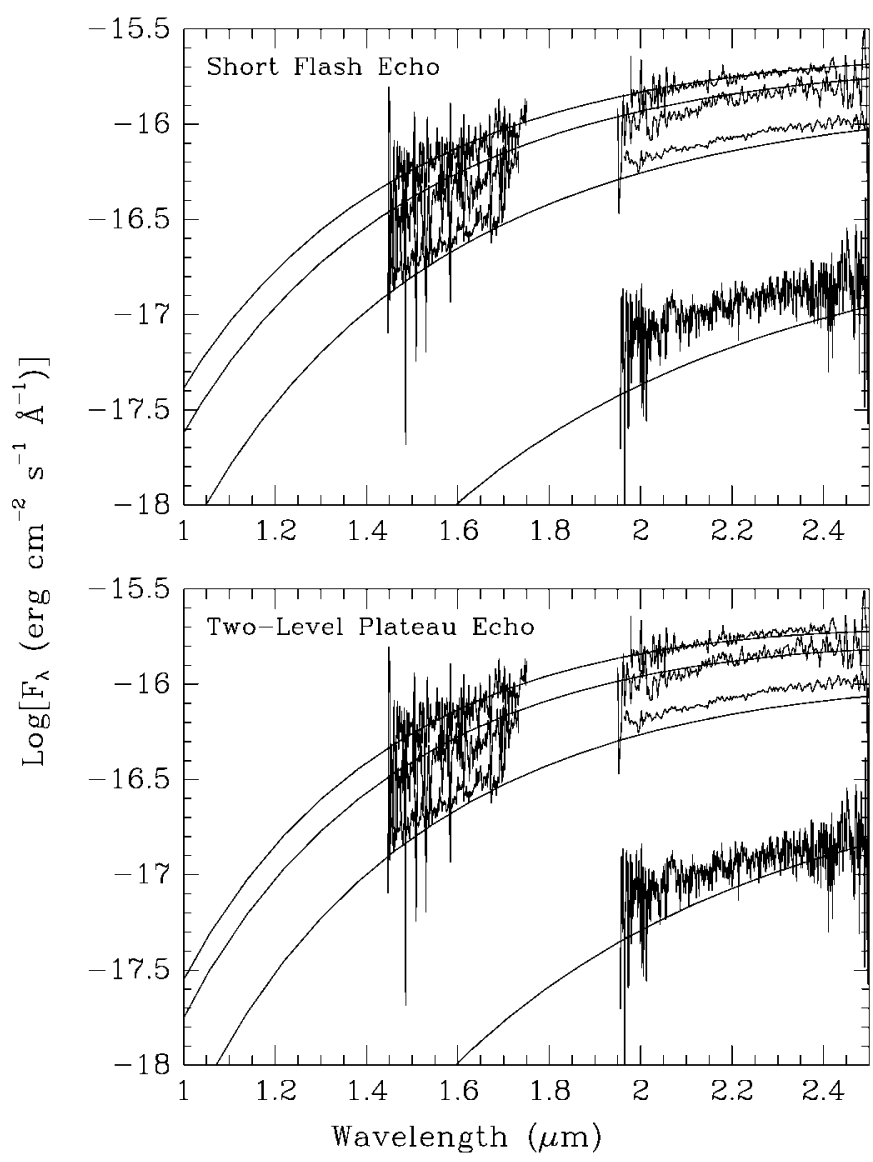

FIG. 3.-Observed NIR spectra of SN 1995N compared to calculated spectra from IR echo models. For the model in the top panel, the input SN light curve was a short exponential decay. The model in the bottom panel results from a two-level top-hat model with a long bright plateau followed by a fainter constant source. The epochs of the observed data are 640, 1078, 1319 , and 2435 days, from top to bottom.

spectral evolution compares well with the observations, although the model luminosity fades more quickly. This is in contrast to the plot in the bottom left panel of Figure 2, where the model fades more slowly than the observed IR luminosity. Again, the reason for the discrepancy is that the observed IR luminosities are only extrapolations based on the $H-K$ temperature fits and are therefore missing a significant component of emission from cool dust. Since the spectral evolution provides a much more direct test, we use this as our primary model discriminant.

While the short-flash echo model is successful enough to suggest that the IR echo scenario may be correct, it involves an unrealistic SN light curve. This model describes an echo in which most of the SN energy is released quickly, in this case within the first 100 days. This timescale, while typical for most supernovae, is not really a good description of a Type IIn supernova such as SN 1995 N. SN 1995 N was observed to have a very slowly decaying light curve (Baird et al. 1998), a feature seen in several other Type IIn events (SN 1987F, SN 1978K, SN 1988Z: Filippenko 1997; SN 1997ab: Hagen et al. 1997). Also, bright X-ray emission was observed in SN $1995 \mathrm{~N}$ at very late times, even beyond the beginning of the observed IR decline. Both of these facts suggest that a much longer timescale should be used for the light source of the echo model.
A longer source light curve is also easier to reconcile with the very large IR luminosity at late time. A simple interpolation of the observed IR luminosities shown in Figure 2 yields a total output of about $5 \times 10^{49}$ ergs in between days 730 and 2435. Integration of the model light curve yields a value closer to $10^{50} \mathrm{ergs}$. Thus, the energy released in the IR is significantly larger than a typical $\sim 100$ day supernova flash but is perhaps consistent with the long-lasting interaction powered emission seen in other bright Type IIn supernovae. Aretxaga et al. (1999) found a lower limit of $2 \times 10^{51}$ ergs for the emission from SN 1988Z during $8.5 \mathrm{yr}$ of observation. Similar emission from SN 1995N would certainly provide enough energy to power the observed dust emission but would again call for a much longer light curve for the echo model.

\subsubsection{CSM Interaction Echo Model}

While a short-flash model is relatively successful at matching the observed NIR spectral evolution, the input SN light curve was not a satisfactory description of SN $1995 \mathrm{~N}$, and we therefore examined echo models with longer supernova light curves. The late-time X-ray flux appears to be relatively constant, thereby making a long top-hat light curve an attractive possibility. For a long top-hat model, the IR continuum continues to rise until after the SN light source shuts off. If the cutoff occurs prior to $t_{v}$, then the IR luminosity levels off until after $t_{v}$ and then begins to fade. Conversely, if the cutoff epoch is after $t_{v}$, then the IR flux continues to rise until the light source shuts off, and then the IR flux quickly decays.

However, what we observe in SN $1995 \mathrm{~N}$ is not consistent with such a model. The NIR flux faded very slowly prior to the $t \approx 1000$ days kink and fairly rapidly thereafter, with no sign of an X-ray cutoff $\approx 200$ days after the IR kink. Furthermore, the qualitative results from the long top-hat model hold true for any fairly flat input light curve. Thus, the rapid decline of the IR flux after the kink forces us to the conclusion that most of the total emission from SN 1995N was released well before $t=1000$ days, even though the late-time $\mathrm{X}$-ray emission was still quite bright.

This suggests a two-component light curve, which we model as a top hat plus constant late-time emission. The results of such a model are plotted in the bottom half of Figure 3. For the model shown, the absorption/emission exponent $n=2$, and the echo timescale parameter $t_{v}=800$ days, which implies a dust-free cavity radius $r_{v} \approx 0.3 \mathrm{pc}$. The model supernova light curve consists of a bright top-hat plateau that lasts for 400 days, followed by constant luminosity emission 20 times fainter than the bright plateau. The fit value of $T_{v} \approx 900 \mathrm{~K}$ for this plot.

Figure 3 shows that this model reproduces the observed IR spectral evolution at least as well as the short-flash echo model. However, where the short-flash model was based on an unsatisfying description of the SN light curve, the twocomponent model is based on a more reasonable approximation for the UV/X-ray light curve of SN 1995N. This shows that the IR excess observed in SN $1995 \mathrm{~N}$ can plausibly be explained as an IR echo. In this case, the echo is not powered by the flash of the supernova at maximum light but rather by the much more slowly evolving emission from the circumstellar interaction. We stress, however, that the particular model fit shown in Figure 3 is probably not unique. 
There are enough parameters that one could likely fit a number of echo models to the data.

Because we did not have nearly as much data from the other Type IIn supernovae, we did not attempt to fit specific echo models for them. But the IR behavior of all of these objects can probably be explained by an interaction echo model. The very large IR luminosities of SN 1997ab and SN $1999 Z$ are easiest to explain with such a model. In SN 1999Z, the rising IR flux seen between days 296 and 465 would suggest that the light source was still emitting a significant amount of energy in this time period. Since we only observed a declining IR flux in SN 1997ab, this would suggest that we caught the IR emission in SN 1997ab after $t_{v}$ in the fading era of the echo.

The behavior of SN 1998S is not suggestive of a spherically symmetric dust echo but is more consistent with an echo from a highly flattened dust distribution. Echo models for flattened dust nebulae with the line of sight near the equatorial plane do predict a bump in the IR light curve near $t_{v}$ (EC88). This would be in line with other evidence for deviations from spherical symmetry in SN 1998S. Spectropolarimetry indicated that both the SN ejecta and the circumstellar environment were aspherical (Leonard et al. 2000; Wang et al. 2001). In addition, a well-resolved triplepeak line profile observed for the hydrogen and helium lines of SN 1998 S was interpreted by Gerardy et al. (2000) as emission from interaction with dense, clumpy circumstellar gas in a ring or disk surrounding the supernova and probably observed from a fairly high inclination.

\section{CONCLUSIONS}

We have presented near-infrared photometry and spectra from five recent Type IIn supernovae that exhibited large late-time IR excesses. The IR excesses in these objects seem to have a much slower evolution than those that have been previously presented in the literature, remaining bright many years after maximum light. The resulting energy release via IR dust emission is quite large, approximately $(0.5-1) \times 10^{50}$ ergs from SN $1995 \mathrm{~N}$. The long IR light curve and large luminosity cannot be reconciled with emission from newly formed dust in the ejecta. In addition, for SN $1995 \mathrm{~N}$ and SN 1998S, the IR luminosity is significantly larger than the late-time X-ray emission, ruling out emission from a shock precursor. IR emission from collisionally excited dust in shocked gas also seems unlikely, as the observed temperatures are fairly high $(\sim 1000 \mathrm{~K})$. Moreover, in SN $1995 \mathrm{~N}$ the IR flux declines rapidly after day 1000 , while the X-ray flux shows little sign of fading, even $\approx 200$ days later.

On the other hand, the data do appear to be consistent with an IR echo of the bright, slowly fading emission from the circumstellar interaction. Simple IR echo models are able to reproduce the observed NIR spectral evolution of SN 1995N, and such a scenario helps explain the slow evolution and large IR luminosities seen in these objects. In principle, detailed studies of the IR echoes of these objects could provide important information about the circumstellar environment of Type IIn supernovae and might lend some insight into their progenitors. In practice, however, in order to provide significant constraints, the supernovae will have to be very well observed, including early-time UV and X-ray observations, as well as good near- and mid-infrared observations of the echo.

We would like to thank the observatory staffs at MDM, FLWO, KPNO, APO, MMT, IRTF, and Subaru for their excellent support, especially Hiroshi Terada, who was enormously helpful during the Subaru observations. C. L. G. and R. A. F.'s research is supported by NSF grant 9876703. K. N. has been supported in part by the Grant-inAid for Scientific Research (07CE2002, 12640233) of the Ministry of Education, Culture, Sports, and Technology in Japan.

\section{REFERENCES}

Aretxaga, I., Benetti, S., Terlevich, R. J., Fabian, A. C., Cappellaro, E., Turatto, M., \& Della Valle, M. 1999 , MNRAS, 309, 343

Baird, M. D., Garnavich, P. M., Schlegel, E. M., Challis, P. M., \& Kirshner, R. P. 1998, BAAS, 30, 1323

Beckwith, S., Evans, N. J., II, Becklin, E. E., \& Neugebauer, G. 1976, ApJ, 208, 390

Bode, M. F., \& Evans, A. 1980, MNRAS, 193, 21

Cao, L., Qiu, Y. L., Qiao, Q. Y., Hu, J. Y., Li, W., \& Filippenko, A. 1999, IAU Circ. 7288, 1

Chugai, N. N., \& Danziger, I. J. 1994, MNRAS, 268, 173

Colgan, S. W., Haas, M. R., Erickson, E. F., Lord, S. D., \& Hollenbach, D. J. 1994, ApJ, 427, 874

Danziger, I. J., Lucy, L. B., Bouchet, P., \& Gouiffes, C. 1991, in Supernovae, ed. S. E. Woosley (New York: Springer), 69

Dwek, E. 1983, ApJ, 274, 175

Dwek, E., Petre, R., Szymkowiak, A., \& Rice, W. L. 1987, ApJ, 320, L27

Dwek, E., et al. 1983, ApJ, 274, 168

Elias, J. H., Matthews, K., Neugebauer, G., \& Soifer, B. T. 1986, BAAS, 18,1016

Emmering, R. T., \& Chevalier, R. A. 1988, AJ, 95, 152 (EC88)

Fassia, A., et al. 2000, MNRAS, 318, 1093

Filippenko, A. V. 1997, ARA\&A, 35, 309

Fox, D. W., et al. 2000, MNRAS, 319, 1154

Fransson, C., et al. 2002, ApJ, 572, 350

Freedman, W. L., et al. 2001, ApJ, 553, 47

Garnavich, P., Noriega-Crespo, A., \& Moro-Martin, A. 1996, IAU Circ., 6314,1

Gehrz, R. D. 1988, ARA\&A, 26, 377

Gerardy, C. L., Fesen, R. A., Höflich, P., \& Wheeler, J. C. 2000, AJ, 119, 2968

Graham, J. R., \& Meikle, W. P. S. 1986, MNRAS, 221, 789
Graham, J. R., et al. 1983, Nature, 304, 709

Hagen, H.-J., Engels, D., \& Reimers, D. 1997, A\&A, 324, L29

Hagen, H.-J., \& Reimers, D. 1997, IAU Circ., 6589, 1

Hanson, M. M., Conti, P. S., \& Reike, M. J. 1996, ApJS, 107, 28

Hanson, M. M., Rieke, G. H., \& Luhman, K. L. 1998, AJ, 116, 1915

Hoffleit, D., \& Jaschek, C. 1982, The Bright Star Catalog (4th ed.; New Haven: Yale Univ. Obs.)

Jha, S., Garnavich, P., Challis, P., Kirshner, R., Calkins, M., \& Zhao, P. 1999, IAU Circ., 7107, 2

Kobayashi, N., et al. 2000, Proc. SPIE 4008, 1056

Kozasa, T., Hasegawa, H., \& Nomoto, K. 1989a, ApJ, 344, 325 1989b, ApJ, 346, L81

$1991, A \& A, 249,474$

Kurucz, R. L. 1994, CD-ROM 18, Solar Abundance Model Atmospheres for $0,1,2,4$ and $8 \mathrm{~km} / \mathrm{s}$ (Cambridge: SAO)

Leonard, D. C., Filippenko, A. V., Barth, A. J., \& Matheson, T. 2000, ApJ, 536,239

Lewis, J. R., et al. 1994, MNRAS, 266, 27

Li, W.-D., Li, C., \& Wan, Z. 1998, IAU Circ., 6829, 1

Livingston, W., \& Wallace, L. 1991, NSO Tech. Rep. 91-001

Lucy, L. B., Danziger, I. J., Gouiffes, C., \& Bouchet, P. 1991, in Supernovae, ed. S. E. Woosley (New York: Springer), 82

McCray, R. 1993, ARA\&A, 31, 175

Merrill, K. M. 1980, IAU Circ., 3444, 3

Osterbrock, D. E. 1989, Astrophysics of Gaseous Nebulae and Active Galactic Nuclei (Mill Valley: University Science Books)

Persson, S. E., Murphy, D. C., Krzeminski, W., Roth, M., \& Rieke, M. J. 1998, AJ, 116, 2475

Pollas, C., Albanese, D., Benetti, S., Bouchet, P., \& Schwarz, H. 1995, IAU Circ., 6170, 1

Pooley, D. et al. 2002, ApJ, 572, 932 
Qiu, Y. L. 1998, IAU Circ., 6835, 1

Rybicki, G. B., \& Lightman, A. P. 1979, Radiative Processes in Astrophysics (New York: Wiley)

Schlegel, D. J., Finkbeiner, D. P., \& Davis, M. 1998, ApJ, 500, 525

Schlegel, E. M. 1990, MNRAS, 244, 269

Schultz, A., et al. 2001, NICMOS Instrument Handbook, Version 4.1 (Baltimore: STScI)
Schwartz, M. 1999, IAU Circ., 7106, 2

Telesco, C., Becklin, E., Koehler, R., \& Gatley, I. 1981, IAU Circ., 3613, 2

Wallace, L., Hinkle, K., \& Livingston, W. 1993, NSO Tech. Rep. 93-001

Wang, L., Howell, D. A., Höflich, P., \& Wheeler, J. C. 2001, ApJ, 550, 1030

Wooden, D. H., Rank, D. M., Bregman, J. D., Witteborn, F. C., Tielens, A. G. G. M., Cohen, M., Pinto, P. A., \& Axelrod, T. S. 1993, ApJS, 88, 477 